\title{
REVIEW ARTICLE OPEN \\ New insights into the cell- and tissue-specificity of glucocorticoid actions
}

\author{
Linda Quatrini (iD) and Sophie Ugolini ${ }^{2}$
}

Glucocorticoids (GCs) are endogenous hormones that are crucial for the homeostasis of the organism and adaptation to the external environment. Because of their anti-inflammatory effects, synthetic GCs are also extensively used in clinical practice. However, almost all cells in the body are sensitive to GC regulation. As a result, these mediators have pleiotropic effects, which may be undesirable or detrimental to human health. Here, we summarize the recent findings that contribute to deciphering the molecular mechanisms downstream of glucocorticoid receptor activation. We also discuss the complex role of GCs in infectious diseases such as sepsis and COVID-19, in which the balance between pathogen elimination and protection against excessive inflammation and immunopathology needs to be tightly regulated. An understanding of the cell type- and context-specific actions of GCs from the molecular to the organismal level would help to optimize their therapeutic use.

Keywords: Glucocorticoids; inflammation; immune response

Cellular \& Molecular Immunology (2021) 18:269-278; https://doi.org/10.1038/s41423-020-00526-2

\section{INTRODUCTION}

Glucocorticoids (GCs) are steroid hormones synthesized and secreted into the bloodstream following HPA (hypothalamicpituitary-adrenal) axis activation. Under physiological conditions, endogenous GCs (cortisol in humans and corticosterone in rodents) are released by the adrenal gland according to a circadian rhythm dictated by light-dark stimuli received by the hypothalamus. These hormones promote adaptation to the external environment by regulating behavioral rhythms, including the sleep-wake and feeding cycles. ${ }^{1}$ The HPA axis is also activated by stressors (threats to physiological or psychological integrity), including infections and inflammatory processes, and GC production contributes to restoring homeostasis. GCs bind to the glucocorticoid receptor (GR), also called $N R 3 C 1$, which is a member of the nuclear receptor superfamily of liganddependent transcription factors. GR is expressed on almost all cell types in the body and regulates the expression of many genes by inducing (transactivation) or inhibiting (transrepression) transcription (Fig. 1). GR target genes are involved in major physiological processes, including metabolic, developmental, cognitive and immune processes, and these pathways are necessary for life after birth. ${ }^{2}$ The potent immunosuppressive and anti-inflammatory properties of GCs have led to their widespread medical use to treat inflammatory disorders (Box 1). GCs were first used to treat rheumatoid arthritis in the 1940s by Dr Philip Hench, who was awarded the Nobel Prize in Physiology or Medicine for this discovery. Many synthetic GCs have since been developed, some of which are more potent than endogenous cortisol. Synthetic GCs have prolonged half-lives and bind with less avidity to serum proteins, thereby diffusing more rapidly into cells. They are currently among the standard treatments for reducing inflammation and immune activation in many inflammatory disorders, including asthma, allergic rhinitis, dermatological, ophthalmic, neurological and autoimmune diseases, and in allotransplantation, sepsis and cancer $^{3-6}$ (Box 1).

However, the therapeutic use of GCs may be limited by the onset of glucocorticoid resistance, which is defined as a poor response to GCs. Such resistance is generally acquired but may, in a small percentage of cases, be congenital due to mutations in the GR-encoding gene. ${ }^{7}$ Moreover, adverse effects associated with GC treatment are frequently reported. These adverse effects result from the actions of GCs on nonimmune physiological processes, such as glucose production in the liver, glucose uptake in skeletal muscle or insulin secretion by pancreatic $\beta$ cells (Box 1).

Gene expression analysis in human peripheral blood mononuclear cells (PBMCs) treated with dexamethasone showed that, in some conditions, GCs can regulate $\sim 20 \%$ of the human genome. ${ }^{8}$ The general mechanisms underlying the control of gene expression by GR have been extensively investigated over the last few decades (Fig. 1) and have been precisely described previously ${ }^{9,10}$ Here, we highlight the many levels of GR-mediated regulation that have been identified so far and may help to predict the effect of GCs from the molecular to the organismal level (Fig. 2). Taking this complexity into account, we also summarize the pathways regulated by endogenous and synthetic GCs in lymphocytes and myeloid cells. Finally, we use sepsis as an example of a pathological condition for which molecular and cellular studies can improve predictions regarding the systemic response to GCs. We stress the need for cell-targeted GC therapy to prevent not only the well-known adverse effects of GCs but also those effects that may reduce treatment efficacy.

\footnotetext{
${ }^{1}$ Department of Immunology, IRCCS Bambino Gesù Children's Hospital, Rome, Italy and ${ }^{2}$ Aix Marseille Univ, CNRS, INSERM, Centre d'Immunologie de Marseille-Luminy, Marseille, France

Correspondence: Linda Quatrini (linda.quatrini@opbg.net) or Sophie Ugolini (ugolini@ciml.univ-mrs.fr)
}

Received: 19 May 2020 Revised: 11 July 2020 Accepted: 31 July 2020

Published online: 31 August 2020 

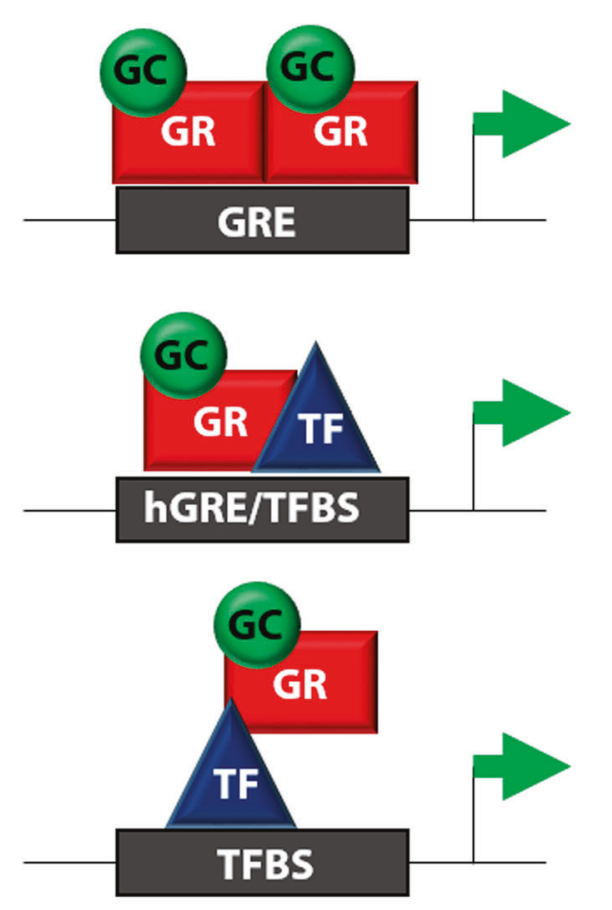
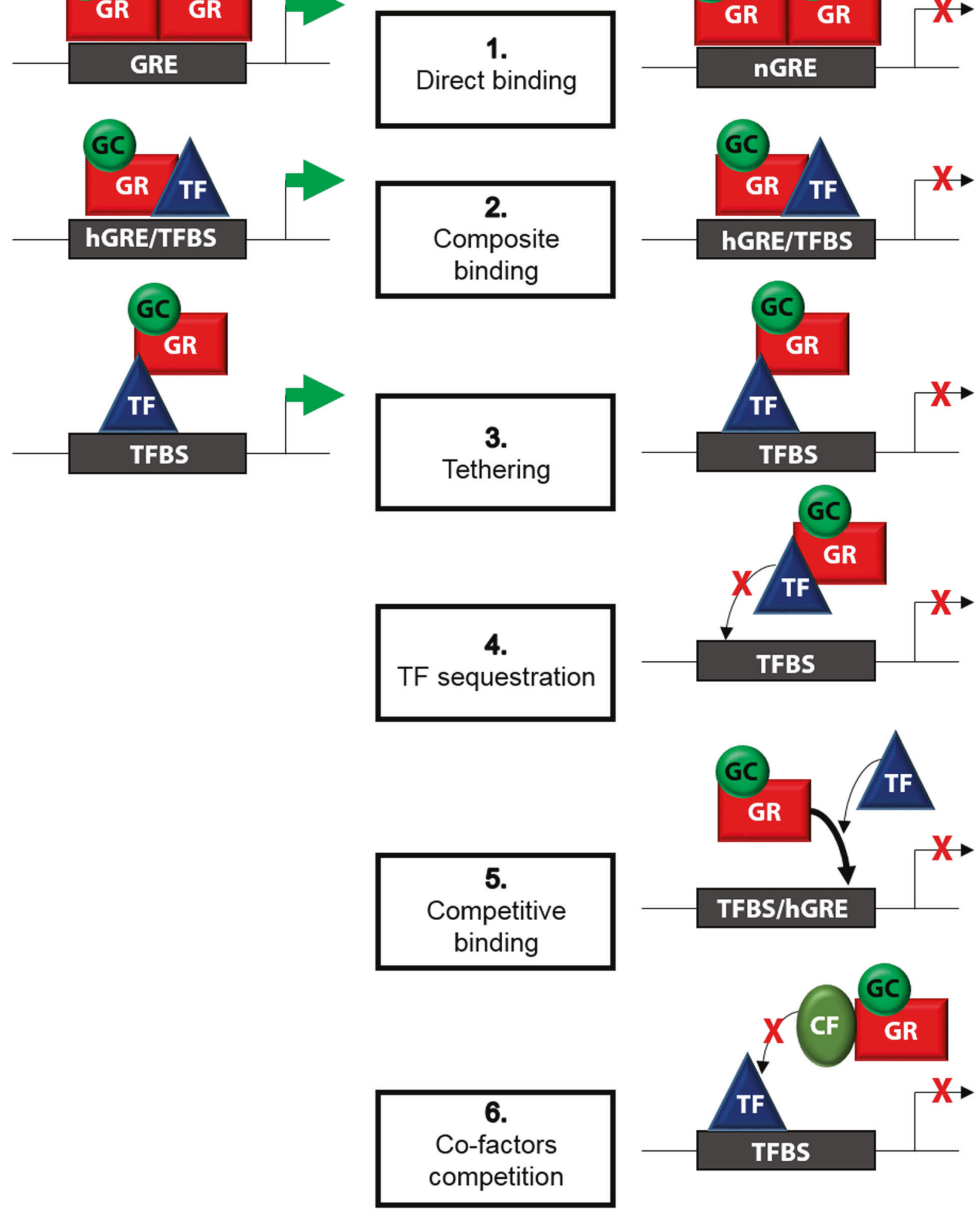

Fig. 1 Genomic signaling mediated by GR. Upon binding to GCs, GR is translocated to the nucleus, where it regulates gene expression by directly binding to DNA or other transcription factors (TFs). (1) The GR homodimer binds to a GC responsive element (GRE) or inverted repeated negative GRE ( $\mathrm{GGRE}$ ), leading to gene transcription or repression, respectively. (2) The GR monomer binds to half the GRE (hGRE) close to the binding site of another TF (TFBS), resulting in gene transcription or repression. Without directly binding to DNA, the GR monomer can physically interact with another TF, thereby influencing its activity (3) or preventing its binding to DNA. (4) GR can also compete with other TFs for binding to DNA (5) or co-factors (CFs) required for transcription (6) 
BOX 1 Therapeutic use of GCs: benefit/risk ratio

GCs can have major therapeutic benefits, but their administration, particularly if prolonged and at high doses, is also associated with severe adverse effects, which constitute the principal factor limiting GC therapy. These adverse effects include metabolic disorders and tissue dysfunction and are linked to the pleiotropic effects of GCs, which act on almost all cell types in the body. GC treatment can also induce skin atrophy and disturb wound healing, as GCs reduce the proliferation of keratinocytes and dermal fibroblasts. ${ }^{102-105}$ Moreover, children on long-term systemic GC therapy commonly experience growth failure and delayed puberty. ${ }^{106}$ GCs also have damaging effects on bones in adults, favoring osteoporosis and increasing fracture risk. ${ }^{107}$ Other adverse effects include hypertension, diabetes, dyslipidemia, hyperglycemia, and adrenal insufficiency leading to iatrogenic Cushing's syndrome. ${ }^{108}$ Another complication of long-term treatment with GCs is GC resistance, resulting in the inability of GCs to exert the desired effects on target tissues, limiting treatment efficacy. ${ }^{108}$

The clinical situation determines the choice of the most appropriate GC and mode of administration, taking the risk/benefit ratio into account. Clinicians also have to adapt their therapeutic strategies to the individual susceptibilities of patients, which are difficult to predict, and determine the occurrence and severity of adverse effects. ${ }^{109}$ It has become clear that an understanding of how GCs exert their effects in a cell- and tissue-specific manner is crucial for the development of novel therapeutic strategies to maximize the beneficial effects and minimize the adverse effects.

The first attempts to dissociate the desired and undesirable effects of GCs were based on the assumption that these effects were differentially mediated by transactivation and transrepression via GR. ${ }^{110}$ The adverse effects of GC therapy were thought to be induced by dimer-mediated transactivation because the genes induced by this mechanism are involved in glucose synthesis and metabolism, for example. In contrast, the anti-inflammatory effects were mostly attributed to the monomer-mediated transrepression of transcription factors, such as AP-1 and NFKB. These observations led to the search for dissociated GR ligands that selectively induce the formation of GR monomers, known as "SEGRA" (selective GR agonists). ${ }^{110}$

However, despite initial enthusiasm, these synthetic steroids were found to induce adverse effects similar to those observed with classic GC therapy. ${ }^{3,111}$ It was found to be unrealistic to develop ligands that exclusively mediate a single molecular mechanism, and it has become clear that there are many more levels of complexity in the regulation of the $\mathrm{GC}$ response than just transrepression and transactivation One strategy for minimizing adverse effects would involve the delivery of GCs to specific cell types at particular time points during the course of inflammatory disease, but further studies are required to develop such treatments.

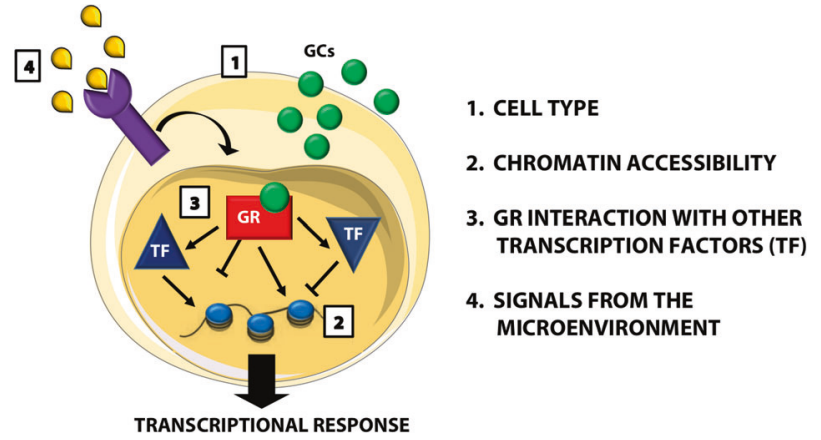

Fig. 2 Factors regulating the transcriptional response to GCs in the cell. The transcriptional program activated in response to GCs results from the complex integration of signals from within and outside the cell. This program depends, first and foremost, on the target cell type and basal expression of target genes. (1) The chromatin accessibility of the promoter regulates transcriptional machinery recruitment. (2) GR activity is also regulated by interactions with other transcription factors (TFs). (3) Finally, the presence of other cells and extracellular molecules determines the microenvironment, (4) which significantly affects the response to GCs

\section{THE HPA AXIS AND THE GC RECEPTOR}

Upon exposure to stress signals or in response to inflammatory cytokines, the hypothalamus releases corticotropin-releasing hormone $(\mathrm{CRH})$, which stimulates the synthesis and secretion of adrenocorticotropic hormone (ACTH) by the pituitary gland. ${ }^{9}$ $\mathrm{ACTH}$ then acts on the adrenal cortex to induce the release of GCs. Once in the bloodstream, GCs exert various effects by binding to

\section{BOX 2 GR isoforms}

Multiple GR isoforms can be produced from the single GR gene by alternative splicing of the nascent transcript, alternative translation initiation of the mature mRNA, and posttranslational modifications of the protein. ${ }^{21}$ These GR subtypes have unique expression and gene regulatory profiles. The GRa isoform mediates GC effects and is highly expressed, whereas the GRB isoform does not bind GCs and exerts weak dominant negative activity on GRa. In addition to their genomic effects, GCs can also exert rapid nongenomic effects mediated by membraneassociated receptors and second messengers. However, the implications of such regulation remain unclear and require further investigation.

GR, which is ubiquitously expressed. In the absence of GCs, GR is located predominantly in the cytoplasm, in a complex containing heat shock proteins and other chaperones. Upon ligand binding, GR undergoes conformational changes, resulting in partial dissociation from the chaperone complex and nuclear translocation. $^{10}$ In the nucleus, GR interacts with DNA and other proteins (Fig. 1). The best described mechanism for the genomic effects of GCs is transactivation, an enhancement of gene expression mediated by the direct binding of GR homodimers to GC response elements (GREs) and the recruitment of coactivators. Inverted negative GREs (nGREs) have been reported to repress gene transcription upon GR binding in a specific manner through the recruitment of corepressors and histone deacetylases via transrepression. ${ }^{11}$ The second mechanism described for the genomic effects of GCs involves the binding of monomeric GR and other transcription factors to DNA, which leads to gene activation or repression. The third mechanism involves physical interactions (tethering) between monomeric GR and other transcription factors without DNA contact to influence the activity of these transcription factors. ${ }^{12}$ This process is considered to be the prevailing mechanism of transrepression. Transrepression can also occur through GR-mediated sequestration of transcription factors and competition for DNA or co-factor binding (Fig. 1). ${ }^{13}$

\section{GR MECHANISM OF ACTION: A COMPLEX SCENARIO}

The anti-inflammatory action of GR depends on its ability to directly repress proinflammatory transcription factors, modulate anti-inflammatory genes, and induce lymphocyte apoptosis. ${ }^{14}$

Upon GC binding, GR inhibits the transcription factors AP-1 (activator protein-1) and NF-KB (nuclear factor kappa B), major downstream effectors of proinflammatory signaling pathways. ${ }^{10} \mathrm{AP}$ 1 is a heterodimer of c-Jun and Fos that acts downstream of the MAPK (mitogen-activated protein kinase) pathway and induces the transcription of many genes involved in inflammatory and immune responses. GR inhibits this pathway by both directly interfering with c-Jun-mediated transcription and inducing MAPK phosphatase 1 (MKP-1), which in turn dephosphorylates and inactivates all components of the MAPK cascade. ${ }^{15,16}$ GR inhibits NF-KB mostly by direct interaction, which prevents NF-KB translocation to the nucleus $^{17}$ and represents a mechanism of transrepression/sequestration (Fig. 1). ${ }^{18,19}$ GR can also inhibit NF-KB indirectly by inducing the expression of TSC22D3, the gene encoding GILZ (glucocorticoidinduced leucine zipper), in almost all cell types. GILZ lacks DNA binding activity but can bind NF-KB and suppress its function. ${ }^{20}$

$\mathrm{GR}$ also modulates gene transcription through direct binding to DNA. However, the presence of GREs in the promoter of a gene is not sufficient for GR to regulate the expression of that gene. The genes targeted by the GR differ among cell types. This functional heterogeneity may be at least partly due to the capacity of cells to generate dozens of $\mathrm{GR}$ isoforms, potentially regulating the expression of specific sets of genes (Box 2). ${ }^{21}$ The cell typespecific activities of GCs depend on many other factors, including the chromatin accessibility of target genes, which modulates the availability of DNA sequences for binding to components of the transcription machinery. The accessibility of chromatin depends on its condensation state, which is regulated by histone and DNA 
modifications. Chromatin accessibility contributes to determining the quality and magnitude of GC responses. ${ }^{22}$ Genome-wide transcriptional responses to GC were recently compared in subsets of human primary hematopoietic and nonhematopoietic cells treated with GCs in vitro. ${ }^{23}$ This study showed that transcriptional responses to GCs were cell type-dependent due to differences in the baseline expression and chromatin accessibility of the target genes (Fig. 2). The study also revealed shared and lineagedependent effects. The pathways that were affected strongly and similarly by GCs in all cell types analyzed were mostly protein kinase-driven signaling cascades, including the MAPK and cytokine receptor signaling pathways. However, some pathways were more strongly affected in hematopoietic cells and others in nonhematopoietic cells. ${ }^{23}$ Cell type-specific responses were also identified. For example, GCs functionally impair BCR (B-cell receptor) and TLR7 (Toll-like receptor 7) signaling and selectively upregulate the immunomodulatory cytokine $\mathrm{IL}-10$ and the terminal differentiation factor BLIMP-1 (B lymphocyte-induced maturation protein-1) in $B$ cells. ${ }^{23}$ These findings were confirmed in $B$ cells that were freshly isolated from healthy volunteers who received a single intravenous dose of methylprednisolone. ${ }^{23}$ These findings may provide a molecular explanation for the efficacy of GC therapy in antibody-mediated autoimmune diseases.

Moreover, the GR-mediated regulation of target genes depends on other transcription factors that are available for binding in the nucleus, as clearly demonstrated by studies of the interplay between GC and TNF signaling. TNF is an important inducer of glucocorticoid resistance. ${ }^{24,25}$ Proteome-wide proximity mapping showed that TNF signaling antagonizes GR activity not by affecting its nuclear translocation, dimerization or ability to bind DNA but by strongly modulating its interactome. ${ }^{26}$ This study clearly demonstrated that the various stimuli from the microenvironment received by the cell reshape the nuclear interactions of GR with its cofactors, altering the final transcriptional outcome (Fig. 2).

Both in vitro and in vivo studies have been used to decipher the precise molecular mechanisms regulating GR activity. In vitro studies are important for identifying the molecular mechanisms of action of GR in one cell type, whereas in vivo studies shed light on the effects of multiple integrated pathways at the whole-body level and in certain physiological and pathological conditions. These two approaches are complementary in addressing the complexity of $G R$ regulation. Early studies on the role of $G R$ in vivo involved the generation of GR-deficient mice. Nr3c1-knockout mice die from respiratory failure within a few hours of birth because of impaired lung development. ${ }^{2}$ These mice have therefore mostly been used for studies of GR functions during embryonic development. The GRdim model, which is characterized by a point mutation impairing dimerization and GRE-dependent transactivation, was then developed $^{27}$ (Fig. 1). This mouse model played an important role in determining which genes were regulated by monomeric versus dimeric GR. GRdim mice are viable, demonstrating the importance of the dimerization-independent functions of the GR involving crosstalk with other transcription factors in vivo. Finally, the Cre/LoxP system has been used to determine the cell- and tissue-specific role of GR in mouse models through specific inactivation of the GR gene in certain cell types. These mouse models made it possible to delineate cell type-specific GR functions and gene targets. ${ }^{28}$

The multiple levels of GC-mediated regulation described above show that, in addition to intrinsic differences between cell types (e.g., baseline expression of target genes and chromatin accessibility), extrinsic factors, including signals from the microenvironment, may affect GR transcriptional regulation (Fig. 2).

\section{GR TARGETS IN LYMPHOCYTES: INHIBITORY CHECKPOINTS ENTER THE SCENE}

GCs have been widely used in the treatment of lymphoproliferative disorders for their proapoptotic effects. Indeed, in the T cell lineage, GCs are known to cause cell death in immature thymocytes, as well as $\mathrm{T}$ cell tumors. ${ }^{29,30}$ However, $\mathrm{T}$ cell sensitivity to GC-induced apoptosis depends on intrinsic cellular factors, such as the degree of activation and the timing of GC exposure (before, during, or after activation). ${ }^{31}$ In addition to cell death, GCs also regulate T cell effector functions by acting on key transcription factors. For example, GR inhibits T-bet by a transrepression mechanism, blocking the ability of T-bet to bind DNA and activate the transcription of target genes, including Ifng. ${ }^{32}$ GR can also interfere with GATA-3 signaling in multiple ways: by reducing chromatin accessibility in the IL-5 gene promoter region, ${ }^{33}$ by competing with GATA-3 for importinalpha interactions and enabling nuclear localization, ${ }^{34}$ and by inhibiting p38 MAPK functions, thus preventing GATA-3 phosphorylation. ${ }^{35}$ The regulation of the Th1/Th2 ratio by GCs has been extensively studied in asthma models, and GC treatment has recently been shown to decrease GATA-3 expression by inhibiting Notch1 signaling, reducing airway responsiveness and inflammation. ${ }^{36}$ In this pathological context, the shift toward Th2 or Th1 responses depends on the length of GC treatment: shortterm treatments may be beneficial, resulting in the inhibition of Th2 cytokine production, while long-term treatments might induce a shift toward Th2 predominance, increasing the severity of the allergy. ${ }^{37}$ Regulation of the Th17 response by GCs is also context-dependent. In patients with psoriasis, for example, Th17 cells are successfully suppressed by GCs, whereas in patients with lupus erythematosus, Th17 cells are refractory to GC treatment. $^{38}$

Stress hormones control leukocyte trafficking and, in particular, GCs control diurnal oscillations in T cell distribution and responses in mice by inducing IL-7R and CXCR4 expression. ${ }^{39} \mathrm{~A}$ recent study also showed that GCs upregulate CXCR4 in mouse B cells and that GR selectively orchestrates $B$ cell migration between bone marrow and blood. ${ }^{40}$ Of note is that in chronic lymphocytic leukemia (CLL) patients, GC administration causes an increase in blood lymphocyte counts, ${ }^{41}$ although the underlying mechanism has not yet been elucidated.

A new immunosuppressive mechanism of action of GCs in cytotoxic lymphocytes has been recently discovered, consisting of the induction of immune checkpoints, which are inhibitory receptors that represent the last revolutionary targets in immunotherapy. The importance of the cellular microenvironment in shaping the GR transcriptional response, including the induction of an immune checkpoint, was demonstrated in a study investigating the in vivo effect of endogenous GCs released upon infection with murine cytomegalovirus (MCMV). ${ }^{42}$ In this model, the gene encoding GR was ablated in lymphocyte subsets expressing NKp46, including natural killer (NK) cells and type 1 innate lymphoid cells (ILC1s). Transcriptomics analysis of the main organs associated with viral replication (spleen and liver) revealed that GC-dependent regulation of gene expression was both cell type- and tissue-dependent. ${ }^{42}$ The gene encoding the inhibitory receptor PD-1 (programmed cell death protein 1) was indeed induced by the GR pathway only in splenic NK cells of infected mice. Thus, endogenous GC-mediated regulation of the host response to pathogens is dependent on the composition of the cell microenvironment and activation status. ${ }^{42}$ In this model, GRinduced PD-1 expression is required to limit immunopathology and promote resistance to viral infection. More recently, it was demonstrated that GCs are present at high levels in the pleural effusions of lung cancer patients and that GCs together with the cytokines present in the tumor microenvironment induce PD-1 expression on human NK cells. ${ }^{43}$ The role of GCs in inducing PD-1 expression can thus be beneficial or detrimental depending on the pathological context (infectious diseases vs cancer). GCs induce PD-1 expression on both mouse and human NK cells, but while this induction occurs mainly at the transcriptional level in mice, in human NK cells, PD-1 expression is also indirectly induced by GCs at the posttranscriptional level. ${ }^{42,43}$ Species-specific differences in the 
effects of GCs can therefore exist, suggesting that caution is needed when translating mouse data to humans.

PD-1 upregulation by GCs was also observed in T cells. ${ }^{44,45}$ In this cell type, TCR engagement is sufficient to induce PD-1 expression, and GCs can further enhance this induction. In particular, the transactivation of PD-1 transcription by GR binding to the PDCD1 promoter was observed in $\mathrm{T}$ cells. ${ }^{45}$ This transcriptional regulation was initially demonstrated in a $T$ cell hybridoma model, but PD-1 was subsequently shown to be upregulated by GCs in primary $T$ cells in vitro and in tumorinfiltrating $\mathrm{CD}^{+} \mathrm{T}$ cells from tumor-bearing mice treated with dexamethasone in vivo. ${ }^{45}$ However, another study showed that corticosteroid treatment did not modify PD-1 expression on $\mathrm{CD}^{+}$ $\mathrm{T}$ cells in vivo. ${ }^{46}$ This discrepancy may be explained by the inoculation of mice with different types of tumors, which induced different microenvironments, or by differences in the GC regimen and dose. However, even if this last study showed no direct effect of GCs on immune checkpoint expression, the findings suggested that corticosteroids could impair the antitumor effects of immune checkpoint blockade. Indeed, in mice challenged with tumors expressing neoantigens, GC treatment inhibited the response and proliferation of low-affinity memory $\mathrm{CD}^{+} \mathrm{T}$ cells by suppressing fatty acid metabolism, which is essential for memory $T$ cells. ${ }^{46}$ Moreover, an analysis of melanoma patients treated by CTLA4 blockade showed that survival rates were reduced for those receiving corticosteroids. ${ }^{46}$

In a mouse model in which GR was selectively ablated in cells expressing Lck, endogenous GCs released upon prolonged psychological stress due to physical confinement were found to upregulate the expression of another immune checkpoint, the inhibitory receptor TIGIT (T cell immunoreceptor with Ig and ITIM domains), on innate-like T cells. ${ }^{47}$

Altogether, these studies may have important implications for the therapeutic use of GCs in patients. The modulation of immune checkpoint receptor expression and/or function in cytotoxic lymphocytes should be more systematically monitored in different pathological contexts. This regulation may have beneficial effects for the treatment of inflammatory diseases/autoimmunity, for example. Conversely, the benefit/risk of GC treatment in cancer patients should be carefully evaluated.

\section{MYELOID CELLS ARE THE MAIN TARGETS OF GCS FOR RESTORING HOMEOSTASIS IN INFLAMMATORY CONDITIONS}

HPA axis activation by inflammatory cytokines is involved in the downregulation of immune responses via a negative feedback loop to restore homeostasis. Monocytes and macrophages are among the most effective producers of proinflammatory mediators. As such, these cells are major targets of GCs for limiting overwhelming and sustained inflammation. In vitro, GCs downregulate the cytokines IL-1 $\beta$, IL-6, IL-12, TNF- $a$, and GM-CSF and the chemokines RANTES, MCP-1, and IL- $8^{48-50}$ in myeloid cells. GCs also inhibit the synthesis of enzymes, such as iNOS and COX-2. ${ }^{51,52}$ In particular, the reduction in iNOS levels is dependent on p38 MAPK dephosphorylation by the GR target MKP-1. ${ }^{53}$ Interestingly, it was recently demonstrated in mouse primary macrophages that the cyclin-dependent kinase 5 (Cdk5) deletion induces the expression of MKP-1. ${ }^{54}$ Therefore, GC-dependent inhibition of iNOS can be enhanced by simultaneously targeting Cdk5. ${ }^{54}$ These data should be confirmed in mouse models to verify whether GCs combined with specific Cdk inhibitors may have a synergistic immunosuppressive effect. Low-dose GCs in combination with Cdk inhibitors may indeed replace high-dose GC therapy, which has severe side effects, in the treatment of inflammatory diseases.

In vivo, the action of GCs has been studied in monocytes, macrophages and granulocytes from mice with myeloid-specific deletion of GR (GR $\left.R^{\text {LysM-Cre }}\right){ }^{55}$ In septic shock models, the lack of control of TNF, IL- 6 and IL-1 $\beta$ production via GR in LysM ${ }^{+}$cells is correlated with increased lethality ${ }^{56,57}$ The role of GR in dendritic cells (DCs) has also been reported in the context of cancer. Moreover, by inducing Tsc22d3 expression, GCs block type I interferon production by DCs, reducing the efficacy of anticancer treatments in subjects under psychological distress. ${ }^{58}$ In mice, the overexpression of $T s c 22 d 3$ via a transgene in DCs abolished the efficacy of immunogenic chemotherapy, whereas conditional knockout of $T s c 22 d 3$ in DCs was sufficient to abrogate the GC-induced impairment in the response to immunogenic chemotherapy. ${ }^{58}$

Even if GCs are mainly used as immunosuppressive agents, their effects can be very complex. As already mentioned, GR can bind many different target genes and transcription factors, and GCs can also exert proinflammatory effects in some conditions. ${ }^{9,59,60}$ For example, GCs can prime macrophages for the immediate early response by inducing the tetraspan protein MS4A4A, which is important for signaling and localization in lipid rafts associated with the N-glycan receptor Dectin $1 .^{61}$ This regulatory process was essential in vivo for dectin-1-dependent activation of NK cells and resistance to metastasis. ${ }^{61}$ Moreover, MS4A4A transcript levels were shown to increase in circulating monocytes from Grave's disease patients upon acute exposure to methylprednisolone. ${ }^{61}$ Therefore, it is now clear that GCs represent a specific "alert" signal for immune cells rather than a general "shut down" signal. ${ }^{62,63}$

Other studies on the effect of GCs on myeloid cells have suggested that the release of endogenous GCs during inflammation is also an important mechanism that shifts the amplification phase of inflammation toward resolution. A study of global gene expression in GC-treated human monocytes revealed that GCs not only suppress the inflammatory functions of monocytes but also induce a shift to an anti-inflammatory activated phenotype, including enhanced phagocytic properties, migratory behavior and protection against apoptosis. ${ }^{64}$ Moreover, GCs have been shown to induce IL-10 production by human monocytes ${ }^{65}$ and murine primary peritoneal and bone marrow macrophages. ${ }^{66}$ In a model of colitis induced by dextran sulfate sodium (DSS) using the $\mathrm{GR}^{\text {LysM-Cre }}$ mouse model, it was shown that GR signaling in myeloid cells is required to resolve inflammation. ${ }^{67} \mathrm{GCs}$ also upregulate the expression of CD163, a scavenger receptor that is a marker of alternatively activated macrophages, in human monocytes and macrophages ${ }^{68,69}$ and increase the ability of human macrophages to engulf apoptotic cells via phagocytosis, thereby contributing to the resolution of inflammation. ${ }^{70,71}$

GCs can also affect antigen presentation. High cortisol levels in patients with sepsis are associated with the downregulation of major histocompatibility complex (MHC) class II expression on circulating monocytes. ${ }^{72}$ GC-induced MHC II downregulation has also been observed in murine macrophages in vitro ${ }^{73}$ and can reduce the capacity of monocytes to induce effector T cell activation.

These findings demonstrate that GCs stimulate monocytes/ macrophages to optimize the clearance of cellular debris and dying neutrophils, leading to the resolution of inflammation. ${ }^{74}$ The effects of GCs on the myeloid cells described above show that specific immunoregulatory mechanisms work together during inflammation, preparing the immune system to respond effectively to a stressor and then shutting down the immune response and restoring homeostasis. It is therefore reasonable to assume that the timing of GC treatment in systemic inflammatory diseases (such as sepsis) may be a crucial determinant of clinical outcome.

\section{GC REGULATION FROM THE MOLECULAR TO THE ORGANISMAL LEVEL: THE EXAMPLE OF SEPSIS}

Host resistance to infection requires a delicate balance between resistance mechanisms that induce pathogen elimination and tolerance mechanisms that protect the tissues. GCs play a key role in controlling this balance. Sepsis is a life-threatening condition characterized by exaggerated and protracted systemic inflammation in response to infection. ${ }^{75}$ Sepsis can lead to severe tissue injury, 
maladaptive repair of vital organs and, eventually, multiple organ failure and death. ${ }^{75}$ As mentioned previously, some inflammatory cytokines released upon infection, including IL-6, TNF-a, and IL-1, activate the HPA axis and induce the production of endogenous GCs. ${ }^{76,77}$ This pathway has been shown to be protective in mouse models of sepsis. ${ }^{78}$ Sepsis is commonly studied in vivo by injecting mice with LPS (lipopolysaccharide) to mimic infection with Gramnegative bacteria. The injection of a single high dose of LPS leads to the overproduction of inflammatory cytokines, which play important roles in mounting effective immune responses to pathogens but may nevertheless cause death by mimicking the hyperinflammation observed during sepsis. However, mice exposed to sublethal doses of LPS are more resistant to a subsequent lethal LPS challenge. ${ }^{79}$ This treatment induces a temporary state of hyporesponsiveness in the innate immune system, which is associated with increased endotoxin tolerance. ${ }^{80-83}$ This endotoxin tolerance process plays a key role in protecting the host against the deleterious effects of the excessive inflammatory response observed during septic shock. However, this tolerance also induces a refractory state that can increase host susceptibility to infection with other pathogens. A similar refractory state associated with endotoxin tolerance in patients with sepsis has been observed in noninfectious systemic inflammatory response syndrome (SIRS) caused by trauma, surgery or cardiac arrest. ${ }^{84}$

Recent studies in mice with selective deletion of GR in immune cell subsets have dissected the role of GC regulation in establishing the delicate balance between pathogen elimination and tolerance mechanisms to prevent excessive tissue damage. ${ }^{85,86}$ Studies of GR deletion in DCs revealed that GC signaling in DCs downregulated IL-12 production, protecting against LPS-induced septic shock by reducing inflammatory cytokine production, hypothermia, and mortality. ${ }^{86}$ Moreover, this study showed that this signaling pathway is responsible for the establishment of endotoxin tolerance, which is characterized by the reprogramming of myeloid cells toward an anti-inflammatory phenotype. The role of GC signaling in endotoxin tolerance has also been investigated in NK cells. ${ }^{85}$ It was shown that the endogenous GCmediated inhibition of IFN $y$ production in $\mathrm{NKp}^{+} 6^{+}$cells is required for the development of endotoxin tolerance. This study revealed that endogenous GCs act on NK cells, decreasing their IFNY production. This regulatory pathway indirectly favors the establishment of an immunosuppressive state characterized by high serum concentrations of the anti-inflammatory cytokine IL- $10 .{ }^{85}$

No pharmacological treatment with proven efficacy is currently available for sepsis, other than appropriate antibiotic agents, fluids and vasopressors as needed. Synthetic steroids have been used as an adjuvant therapy for septic shock for more than 40 years. However, uncertainties remain about their safety and efficacy, and it is still not clear whether the clinical benefits of GC therapy outweigh the side effects, as clinical trials have yielded conflicting results. ${ }^{75}$ Randomized controlled trials conducted in the 1980s reported an association between the use of high doses of methylprednisolone and rates of morbidity and mortality that were higher than those of untreated controls. ${ }^{87,88}$ Two subsequent randomized controlled trials reported opposite results for the effect of low-dose hydrocortisone on mortality in patients with septic shock, although both studies reported a reversal of shock earlier in patients treated with hydrocortisone than in control subjects. ${ }^{89,90}$ Two recent studies (Adjunctive Corticosteroid Treatment in Critically III Patients with Septic Shock ${ }^{91}$ and Activated Protein $C$ and Corticosteroids for Human Septic Shock ${ }^{92}$ ) constitute the largest comprehensive analyses to date on the effects of hydrocortisone in critically ill patients with septic shock who were treated medically or surgically. These studies reported conflicting results of the effect of GCs on 90day survival, but both studies reported beneficial effects of hydrocortisone on secondary outcomes such as shock reversal and the duration of mechanical ventilation.

Current clinical practice guidelines recommend the use of hydrocortisone in patients with septic shock if adequate fluid resuscitation and vasopressors do not restored hemodynamic stability. ${ }^{93}$ However, despite the highly beneficial effects of GCs in cases of serious systemic inflammation, the sustained administration of these drugs throughout the course of disease can lead to postseptic immunosuppression and a loss of reactivity in circulating leukocytes, which are associated with an increased risk of succumbing to opportunistic infections. ${ }^{94}$ Given the uncertainties about the efficacy of this treatment, specific strategies should be developed for each group of patients.

The data from the in vivo mouse studies described above ${ }^{85,86}$ suggest that agonizing or antagonizing GC actions in specific cell types at particular times during the course of the inflammatory response may prevent shock damage while restoring a functional immune response. For example, GCs could be conjugated to antibodies specific for certain immune cell types. ${ }^{95}$ However, the clinical efficacy of such approaches has yet to be assessed.

\section{GCs IN COVID-19}

A cytokine storm similar to that caused by bacterial sepsis is induced by the novel coronavirus, namely, severe acute respiratory syndrome coronavirus 2 (SARS-CoV2). ${ }^{96}$ This virus has caused a global pandemic since its outbreak in December 2019 in China, leading to a public health emergency that is still ongoing at the time of this review. The disease called Coronavirus disease 2019 (COVID-19) leads to the development of severe respiratory illness in $\sim 20 \%$ of patients, and $5 \%$ of patients require intensive care. Some of the earliest analyses of COVID-19 patients suggested that severe disease and death were dependent not only on the damage induced in the lungs by the virus but also the high levels of cytokines, particularly IL-6, detected in the blood of critically ill patients due to an overreactive immune response. ${ }^{97,98}$ As in sepsis, GC treatment for acute respiratory distress syndrome is questionable because such treatment broadly suppresses the immune system and may actually hamper the body's ability to

BOX 3 Effects of GC therapy on nonimmune cells

Given the pleiotropic effects of GCs, previously unappreciated consequences of GC administration involving nonimmune cells must also be considered when GCs are used in clinical practice. For instance, the beneficial effects of GC treatment in asthma result from not only anti-inflammatory effects on immune cells but also the regulation of nonimmune cell functions. Asthma is one of the most prevalent inflammatory diseases worldwide, and synthetic GCs continue to be among the most effective types of drugs for the long-term control of clinical symptoms. ${ }^{6}$ It has been shown in a mouse model of allergic airway inflammation that immune cell GR expression is dispensable for successful GC therapy. ${ }^{112}$ Bone marrow chimeras and cell-targeted GR knockout were used to show that cells of nonhematopoietic origin were essential for GC-mediated relief from symptoms. In particular, GC signaling in surfactant protein C-producing epithelial cells decreased bronchoconstriction and mucus hyperproduction. ${ }^{112}$

GCs are also widely used to treat cancers. They are used, for example, in metastatic breast cancer, to combat the side effects of chemotherapy and treat the symptoms of advanced cancer. It has recently been suggested that GCs should be used with care in the treatment of patients with breast cancer who have developed cancer-related complications. ${ }^{113}$ Endogenous GC levels have been shown to increase during breast cancer progression, and distant metastases have high levels of GR activity. The transplantation of GC-treated tumor cells in mice leads to an increase in metastatic colonization and worsened survival. This effect is dependent on the direct effects of GC on tumor cells. ${ }^{114}$ Proteomics analysis revealed that $G R$ activation induced signaling networks and protein kinases involved in cancer progression. In particular, the authors found that GR activation led to increased colonization mediated by ROR1 (receptor tyrosine kinase like orphan receptor 1), a kinase responsible for epithelial-mesenchymal transition (EMT) that has been implicated in metastasis development. ${ }^{114}$ This study also revealed that GR activation decreases the efficacy of paclitaxel, a chemotherapeutic drug widely used to treat breast cancer. The authors were able to exclude the possibility that the hormones were being synthesized by the cancer cells themselves and to demonstrate the activation of the HPA axis in the context of cancer development. ${ }^{113}$ Overall, these findings suggest that efforts should be made to determine whether stress hormones can affect metastasis and the treatment response in tumors other than breast cancer. In such conditions, antagonizing the effects of GR on tumor cells may be more efficient than treating patients with systemic corticosteroids to prevent metastasis. 


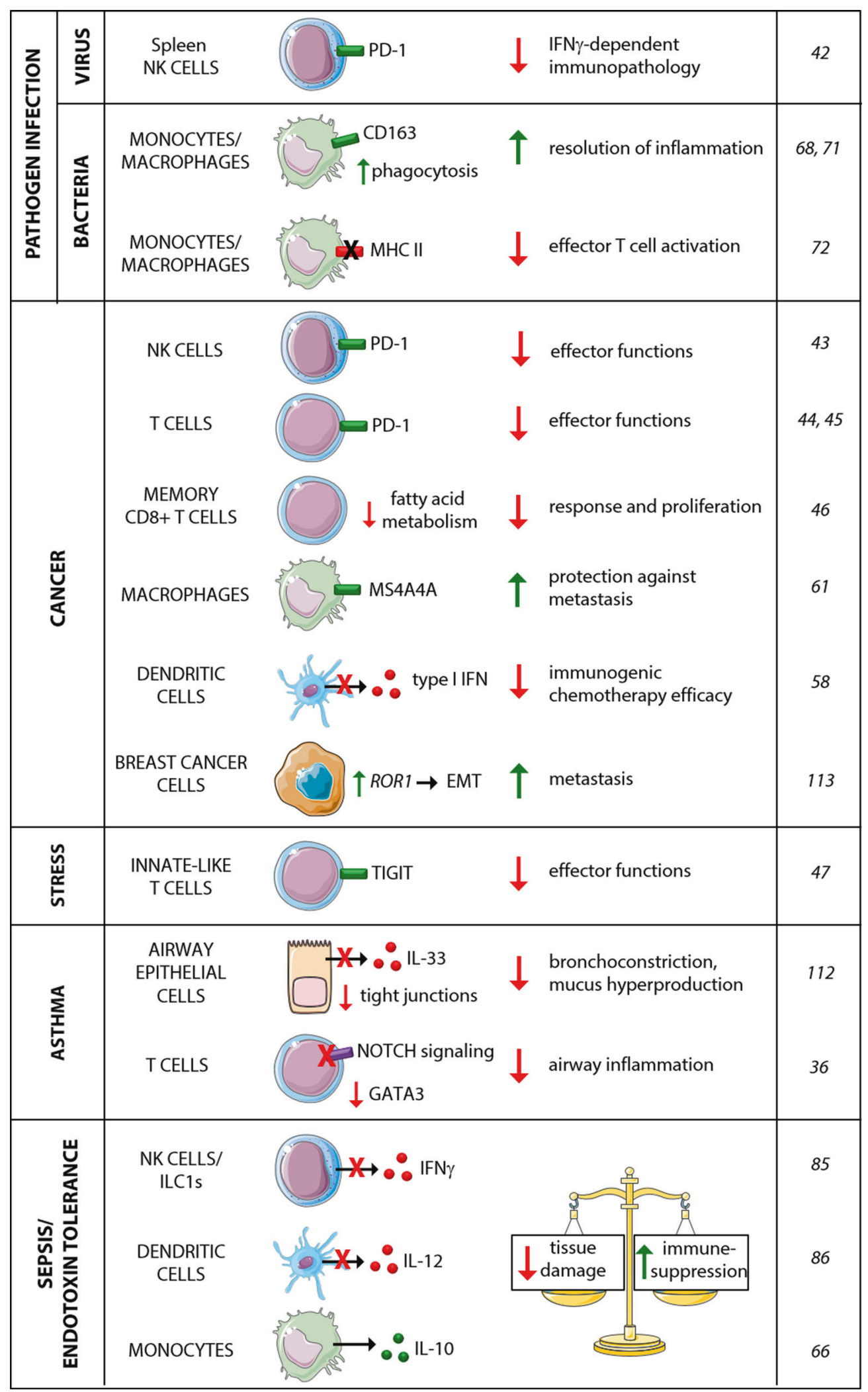

Fig. 3 New targets for GCs in immune and nonimmune cells. Recently identified target molecules of GR are shown. The cell- and contextspecificity of GC regulation is mediated by the pathological condition and the cell type in which the GC targets are located. The consequences of such regulation, which are dependent on context, and the reference numbers are also reported 
keep the virus in check. Nevertheless, given the widespread availability of dexamethasone and some promising results from steroid treatment in previous outbreaks, it was considered important to test this treatment in a rigorous clinical trial. The Randomized Evaluation of COVid-19 thERapY (RECOVERY) trial launched in March 2020 is one of the world's largest randomized controlled trials for coronavirus treatments, testing a range of potential therapies. Over 11,500 patients were enrolled from over $175 \mathrm{NHS}$ hospitals in the United Kingdom. The dexamethasone arm enrolled 2100 participants who received the drug at a low/ moderate dose of $6 \mathrm{mg} /$ day for 10 days, compared to $\sim 4300$ people who received standard care for coronavirus infection. Professor Peter Horby and Professor Martin Landray, chief investigators of the RECOVERY study, announced their findings in a press release on 16 June 2020. The most striking result was that dexamethasone reduced deaths by approximately one-third in critically ill patients requiring ventilation. Importantly, no evidence of benefit was found for patients who did not require oxygen and in those who did not need hospitalization. This pattern of response to dexamethasone confirms that a hyperactive immune response contributes more to morbidity and mortality than the direct effect of the virus in the more severe forms of the disease. Indeed, people infected with SARS-CoV2 do not show symptoms until several days after infection, when collateral damage from the immune response often contributes to the illness. ${ }^{99}$ These results are very important for public health because dexamethasone is the first drug that has been shown to improve survival in COVID-19 patients, and it is also highly available and affordable. In addition, in the context of this pandemic, the benefits of GC treatment in a particular group of patients confirm that it is extremely important to design GC therapies that allow for balance between suppressing the overactive immune response and the spread of the viral infection.

\section{CONCLUDING REMARKS}

The therapeutic anti-inflammatory effects of GCs have been known and used for more than 70 years, but deciphering the complexity of the organism's response to GCs remains challenging. The final outcome is a combination of the different and sometimes opposite responses of the various cell types (Box 3 and Fig. 3), which depend on specific transcriptional programs resulting from complex signal integration. Together, these variables make it very difficult to predict the therapeutic effects of GCs in a given pathological condition. Recent studies that took advantage of more specific experimental approaches than those in the past have contributed to unraveling this complexity. ${ }^{28}$ These studies showed that the molecular response to GCs was cell type- and tissue-specific and was regulated by the cellular microenvironment, which could shape the GR interactome. $^{26,42}$ These findings challenge the common clinical practice of administering corticosteroids to patients with any type of inflammatory disorder. For example, inhibitory checkpoints have been shown to be novel targets in cytotoxic lymphocytes. ${ }^{42-45,47}$ This discovery opens the question of whether GC treatment is beneficial or deleterious for the treatment of cancer patients. Retrospective or prospective studies are thus needed to determine whether corticosteroid administration interferes with immunotherapy in cancer. Moreover, it was shown that GC treatment decreases the inflammatory response responsible for tissue damage in the context of sepsis, which can lead to immunosuppression. ${ }^{85,86}$ Endotoxin tolerance is principally mediated by myeloid cells, and so GR blockade in these cell types after sepsis might restore a functional immune response and protect patients from subsequent infections.

The results of these recent studies suggest that the delivery of GCs to specific target cells and at specific time points during the course of inflammatory diseases may be an efficient therapeutic strategy and may replace the current practice of administering systemic corticosteroids. Developing this promising new approach may increase treatment efficacy and decrease the adverse effects of these drugs. In the context of rheumatoid arthritis, for example, an attempt to improve this therapeutic balance was made by developing "liposomal GCs". Liposomes are organic nanoparticles composed of a phospholipid bilayer, in which synthetic GCs can be enclosed. It was shown in rat and mouse models that liposomal GCs preferentially accumulate in inflamed joints, thus inducing remission in experimental arthritis. ${ }^{100,101}$ Although promising, this approach may be efficient for delivering GCs to the site of inflammation, but it would not be possible to dissociate the effects of GCs on immune and nonimmune cells. Targeted delivery could be achieved, for example, by generating antibody- or peptide-GC conjugates ${ }^{95}$ and should be context-dependent and specific for the time and nature of the inflammatory response. The basis for the development of such new compounds and their use in clinical practice will require deeper investigation of the molecular and cellular actions of GCs. Further studies are therefore required to decipher the cellular and context-specific mechanisms of GC regulation, optimize and possibly target corticosteroid therapy and increase its efficacy while limiting adverse effects.

\section{ACKNOWLEDGEMENTS}

The SU laboratory received funding from the European Research Council (ERC) under the European Union's Horizon 2020 Research and Innovation Program under grant agreement no. 648768, the Agence Nationnale de la Recherche (ANR) (No. ANR-14CE14-0009-01), and the ARC foundation (No. PGA120140200817). The SU laboratory is also supported by institutional grants from INSERM, CNRS, Aix-Marseille University and Marseille-Immunopole to the CIML. L.Q. has received funding from AIRC and from the European Union's Horizon 2020 Research and Innovation Program under the Marie Skłodowska-Curie grant agreement no. 800924.

\section{ADDITIONAL INFORMATION}

Competing interests: The authors declare no competing interests.

\section{REFERENCES}

1. Oster, $\mathrm{H}$. et al. The functional and clinical significance of the 24-hour rhythm of circulating glucocorticoids. Endocr. Rev. 38, 3-45 (2017).

2. Cole, T. J. et al. Targeted disruption of the glucocorticoid receptor gene blocks adrenergic chromaffin cell development and severely retards lung maturation. Genes Dev. 9, 1608-1621 (1995).

3. Vandewalle, J., Luypaert, A., De Bosscher, K. \& Libert, C. Therapeutic mechanisms of glucocorticoids. Trends Endocrinol. Metab. 29, 42-54 (2018).

4. Frei, E. 3rd et al. The effectiveness of combinations of antileukemic agents in inducing and maintaining remission in children with acute leukemia. Blood 26, 642-656 (1965).

5. Herr, I. \& Pfitzenmaier, J. Glucocorticoid use in prostate cancer and other solid tumours: implications for effectiveness of cytotoxic treatment and metastases. Lancet Oncol. 7, 425-430 (2006)

6. Holgate, S. T. \& Polosa, R. Treatment strategies for allergy and asthma. Nat. Rev. Immunol. 8, 218-230 (2008).

7. Dendoncker, K. \& Libert, C. Glucocorticoid resistance as a major drive in sepsis pathology. Cytokine growth factor Rev. 35, 85-96 (2017).

8. Galon, J. et al. Gene profiling reveals unknown enhancing and suppressive actions of glucocorticoids on immune cells. FASEB J. 16, 61-71 (2002).

9. Cain, D. W. \& Cidlowski, J. A. Immune regulation by glucocorticoids. Nat. Rev. Immunol. 17, 233-247 (2017).

10. Kadmiel, M. \& Cidlowski, J. A. Glucocorticoid receptor signaling in health and disease. Trends Pharmacol. Sci. 34, 518-530 (2013).

11. Meijsing, S. H. et al. DNA binding site sequence directs glucocorticoid receptor structure and activity. Science 324, 407-410 (2009).

12. Hudson, W. H., Youn, C. \& Ortlund, E. A. The structural basis of direct glucocorticoid-mediated transrepression. Nat. Struct. Mol. Biol. 20, 53-58 (2013).

13. Timmermans, S., Souffriau, J. \& Libert, C. A general introduction to glucocorticoid biology. Front. Immunol. 10, 1545 (2019).

14. Rhen, T. \& Cidlowski, J. A. Antiinflammatory action of glucocorticoids-new mechanisms for old drugs. N. Engl. J. Med. 353, 1711-1723 (2005).

15. Kassel, O. et al. Glucocorticoids inhibit MAP kinase via increased expression and decreased degradation of MKP-1. EMBO J. 20, 7108-7116 (2001). 
16. Lasa, M., Abraham, S. M., Boucheron, C., Saklatvala, J. \& Clark, A. R. Dexamethasone causes sustained expression of mitogen-activated protein kinase (MAPK) phosphatase 1 and phosphatase-mediated inhibition of MAPK p38. Mol. Cell. Biol. 22, 7802-7811 (2002).

17. Widen, C., Gustafsson, J. A. \& Wikstrom, A. C. Cytosolic glucocorticoid receptor interaction with nuclear factor-kappa B proteins in rat liver cells. Biochemical J. 373, 211-220 (2003).

18. De Bosscher, K. et al. Glucocorticoids repress NF-kappaB-driven genes by disturbing the interaction of p65 with the basal transcription machinery, irrespective of coactivator levels in the cell. Proc. Natl Acad. Sci. USA 97, 3919-3924 (2000).

19. De Bosscher, K., Vanden Berghe, W. \& Haegeman, G. The interplay between the glucocorticoid receptor and nuclear factor-kappaB or activator protein-1: molecular mechanisms for gene repression. Endocr. Rev. 24, 488-522 (2003).

20. Berrebi, D. et al. Synthesis of glucocorticoid-induced leucine zipper (GILZ) by macrophages: an anti-inflammatory and immunosuppressive mechanism shared by glucocorticoids and IL-10. Blood 101, 729-738 (2003).

21. Oakley, R. H. \& Cidlowski, J. A. Cellular processing of the glucocorticoid receptor gene and protein: new mechanisms for generating tissue-specific actions of glucocorticoids. J. Biol. Chem. 286, 3177-3184 (2011).

22. John, S. et al. Chromatin accessibility pre-determines glucocorticoid receptor binding patterns. Nat. Genet. 43, 264-268 (2011).

23. Franco, L. M. et al. Immune regulation by glucocorticoids can be linked to cell type-dependent transcriptional responses. J. Exp. Med. 216, 384-406 (2019).

24. Dejager, L. et al. Neutralizing TNFalpha restores glucocorticoid sensitivity in a mouse model of neutrophilic airway inflammation. Mucosal Immunol. 8, 1212-1225 (2015).

25. Van Bogaert, T. et al. Tumor necrosis factor inhibits glucocorticoid receptor function in mice: a strong signal toward lethal shock. J. Biol. Chem. 286, 26555-26567 (2011).

26. Dendoncker, K. et al. TNF-alpha inhibits glucocorticoid receptor-induced gene expression by reshaping the GR nuclear cofactor profile. Proc. Natl Acad. Sci. USA 116, 12942-12951 (2019).

27. Reichardt, H. M. et al. DNA binding of the glucocorticoid receptor is not essential for survival. Cell 93, 531-541 (1998).

28. Whirledge, S. \& DeFranco, D. B. Glucocorticoid signaling in health and disease: insights from tissue-specific GR knockout mice. Endocrinology 159, 46-64 (2018).

29. Wyllie, A. H. Glucocorticoid-induced thymocyte apoptosis is associated with endogenous endonuclease activation. Nature 284, 555-556 (1980).

30. Strehl, C., Ehlers, L., Gaber, T. \& Buttgereit, F. Glucocorticoids-all-rounders tackling the versatile players of the immune system. Front. Immunol. 10, 1744 (2019).

31. Zacharchuk, C. M., Mercep, M., Chakraborti, P. K., Simons, S. S. Jr. \& Ashwell, J. D. Programmed T lymphocyte death. Cell activation- and steroid-induced pathways are mutually antagonistic. J. Immunol. 145, 4037-4045 (1990).

32. Liberman, A. C. et al. The activated glucocorticoid receptor inhibits the transcription factor T-bet by direct protein-protein interaction. FASEB J. 21, 1177-1188 (2007).

33. Jee, Y. K. et al. Repression of interleukin- 5 transcription by the glucocorticoid receptor targets GATA3 signaling and involves histone deacetylase recruitment. J. Biol. Chem. 280, 23243-23250 (2005).

34. Maneechotesuwan, K. et al. Suppression of GATA-3 nuclear import and phosphorylation: a novel mechanism of corticosteroid action in allergic disease. PLoS Med. 6, e1000076 (2009).

35. Liberman, A. C., Druker, J., Refojo, D., Holsboer, F. \& Arzt, E. Glucocorticoids inhibit GATA-3 phosphorylation and activity in T cells. FASEB J. 23, 1558-1571 (2009).

36. $\mathrm{Hu}, \mathrm{C}$. et al. Glucocorticoids modulate Th1 and Th2 responses in asthmatic mouse models by inhibition of Notch1 signaling. Int. Arch. allergy Immunol. 175, 44-52 (2018)

37. Elenkov, I. J. Glucocorticoids and the Th1/Th2 balance. Ann. N. Y. Acad. Sci. 1024 138-146 (2004)

38. Banuelos, J., Cao, Y., Shin, S. C. \& Lu, N. Z. Immunopathology alters Th17 cell glucocorticoid sensitivity. Allergy 72, 331-341 (2017).

39. Shimba, A. et al. Glucocorticoids drive diurnal oscillations in T cell distribution and responses by inducing interleukin-7 receptor and CXCR4. Immunity 48, 286-298.e6 (2018).

40. Cain, D. W. et al. Murine glucocorticoid receptors orchestrate B cell migration selectively between bone marrow and blood. J. Immunol. 205, 619-629 (2020).

41. Burger, J. A. \& Montserrat, E. Coming full circle: 70 years of chronic lymphocytic leukemia cell redistribution, from glucocorticoids to inhibitors of B-cell receptor signaling. Blood 121, 1501-1509 (2013).

42. Quatrini, L. et al. Endogenous glucocorticoids control host resistance to viral infection through the tissue-specific regulation of PD-1 expression on NK cells. Nat. Immunol. 19, 954-962 (2018).
43. Quatrini, L. et al. Glucocorticoids and the cytokines IL-12, IL-15 and IL-18 present in the tumor microenvironment induce PD-1 expression on human Natural Killer cells. J. Allergy clinical Immunol. https://doi.org/10.1016/j.jaci.2020.04.044 (2020).

44. Xing, K., Gu, B., Zhang, P. \& Wu, X. Dexamethasone enhances programmed cell death 1 (PD-1) expression during $T$ cell activation: an insight into the optimum application of glucocorticoids in anti-cancer therapy. BMC Immunol. 16, 39 (2015).

45. Maeda, N. et al. Glucocorticoids potentiate the inhibitory capacity of programmed cell death 1 by up-regulating its expression on T cells. J. Biol. Chem. 294, 19896-19906 (2019).

46. Tokunaga, A. et al. Selective inhibition of low-affinity memory CD8(+) T cells by corticosteroids. J. Exp. Med. 216, 2701-2713 (2019).

47. Rudak, P. T. et al. Stress-elicited glucocorticoid receptor signaling upregulates TIGIT in innate-like invariant T lymphocytes. Brain, Behav., Immun. 80, 793-804 (2019).

48. $\mathrm{Ma}, \mathrm{W}$. et al. Dexamethasone inhibits IL-12p40 production in lipopolysaccharidestimulated human monocytic cells by down-regulating the activity of c-Jun Nterminal kinase, the activation protein-1, and NF-kappa B transcription factors. J. Immunol. 172, 318-330 (2004).

49. Larsson, S. \& Linden, M. Effects of a corticosteroid, budesonide, on production of bioactive IL-12 by human monocytes. Cytokine 10, 786-789 (1998).

50. Visser, J. et al. Differential regulation of interleukin-10 (IL-10) and IL-12 by glucocorticoids in vitro. Blood 91, 4255-4264 (1998).

51. Di Rosa, M., Radomski, M., Carnuccio, R. \& Moncada, S. Glucocorticoids inhibit the induction of nitric oxide synthase in macrophages. Biochem. Biophys. Res. Commun. 172, 1246-1252 (1990).

52. Lee, S. H. et al. Selective expression of mitogen-inducible cyclooxygenase in macrophages stimulated with lipopolysaccharide. J. Biol. Chem. 267, 25934-25938 (1992).

53. Abraham, S. M. et al. Antiinflammatory effects of dexamethasone are partly dependent on induction of dual specificity phosphatase 1. J. Exp. Med. 203, 1883-1889 (2006).

54. Pfander, P., Fidan, M., Burret, U., Lipinski, L. \& Vettorazzi, S. Cdk5 deletion enhances the anti-inflammatory potential of GC-mediated GR activation during inflammation. Front. Immunol. 10, 1554 (2019).

55. Tuckermann, J. P. et al. Macrophages and neutrophils are the targets for immune suppression by glucocorticoids in contact allergy. J. Clin. Investig. 117, 1381-1390 (2007).

56. Bhattacharyya, S., Brown, D. E., Brewer, J. A., Vogt, S. K. \& Muglia, L. J. Macrophage glucocorticoid receptors regulate Toll-like receptor 4-mediated inflammatory responses by selective inhibition of p38 MAP kinase. Blood 109 4313-4319 (2007)

57. Kleiman, A. et al. Glucocorticoid receptor dimerization is required for survival in septic shock via suppression of interleukin-1 in macrophages. FASEB J. 26, 722-729 (2012)

58. Yang, $H$. et al. Stress-glucocorticoid-TSC22D3 axis compromises therapyinduced antitumor immunity. Nat. Med. 25, 1428-1441 (2019).

59. Hermoso, M. A., Matsuguchi, T., Smoak, K. \& Cidlowski, J. A. Glucocorticoids and tumor necrosis factor alpha cooperatively regulate toll-like receptor 2 gene expression. Mol. Cell. Biol. 24, 4743-4756 (2004).

60. Lannan, E. A., Galliher-Beckley, A. J., Scoltock, A. B. \& Cidlowski, J. A. Proinflammatory actions of glucocorticoids: glucocorticoids and TNFalpha coregulate gene expression in vitro and in vivo. Endocrinology 153, 3701-3712 (2012).

61. Mattiola, I. et al. The macrophage tetraspan MS4A4A enhances dectin-1dependent NK cell-mediated resistance to metastasis. Nat. Immunol. 20, 1012-1022 (2019).

62. Irwin, M. R. \& Cole, S. W. Reciprocal regulation of the neural and innate immune systems. Nat. Rev. Immunol. 11, 625-632 (2011).

63. Quatrini, L., Vivier, E. \& Ugolini, S. Neuroendocrine regulation of innate lymphoid cells. Immunological Rev. 286, 120-136 (2018).

64. Ehrchen, J. et al. Glucocorticoids induce differentiation of a specifically activated, anti-inflammatory subtype of human monocytes. Blood 109, 1265-1274 (2007).

65. Mozo, L., Suarez, A. \& Gutierrez, C. Glucocorticoids up-regulate constitutive interleukin-10 production by human monocytes. Clin. Exp. Allergy 34, 406-412 (2004).

66. Tu, G. W. et al. Glucocorticoid attenuates acute lung injury through induction of type 2 macrophage. J. Transl. Med. 15, 181 (2017).

67. Meers, G. K., Bohnenberger, H., Reichardt, H. M., Luhder, F. \& Reichardt, S. D. Impaired resolution of DSS-induced colitis in mice lacking the glucocorticoid receptor in myeloid cells. PLOS ONE 13, e0190846 (2018).

68. Buechler, C. et al. Regulation of scavenger receptor CD163 expression in human monocytes and macrophages by pro- and antiinflammatory stimuli. J. Leukoc. Biol. 67, 97-103 (2000).

69. Vallelian, F. et al. Glucocorticoid treatment skews human monocyte differentiation into a hemoglobin-clearance phenotype with enhanced heme-iron recycling and antioxidant capacity. Blood 116, 5347-5356 (2010). 
70. Giles, K. M. et al. Glucocorticoid augmentation of macrophage capacity for phagocytosis of apoptotic cells is associated with reduced p130Cas expression, loss of paxillin/pyk2 phosphorylation, and high levels of active Rac. J. Immunol. 167, 976-986 (2001).

71. van der Goes, A., Hoekstra, K., van den Berg, T. K. \& Dijkstra, C. D. Dexamethasone promotes phagocytosis and bacterial killing by human monocytes/macrophages in vitro. J. Leukoc. Biol. 67, 801-807 (2000).

72. Le Tulzo, Y. et al. Monocyte human leukocyte antigen-DR transcriptional downregulation by cortisol during septic shock. Am. J. Respir. Crit. Care Med. 169, 1144-1151 (2004).

73. Celada, A., McKercher, S. \& Maki, R. A. Repression of major histocompatibility complex IA expression by glucocorticoids: the glucocorticoid receptor inhibits the DNA binding of the X box DNA binding protein. J. Exp. Med. 177, 691-698 (1993).

74. Yona, S. \& Gordon, S. Inflammation: glucocorticoids turn the monocyte switch. Immunol. Cell Biol. 85, 81-82 (2007).

75. Cecconi, M., Evans, L., Levy, M. \& Rhodes, A. Sepsis and septic shock. Lancet 392, 75-87 (2018).

76. Perlstein, R. S., Whitnall, M. H., Abrams, J. S., Mougey, E. H. \& Neta, R. Synergistic roles of interleukin-6, interleukin-1, and tumor necrosis factor in the adrenocorticotropin response to bacterial lipopolysaccharide in vivo. Endocrinology 132, 946-952 (1993).

77. Rivier, C., Chizzonite, R. \& Vale, W. In the mouse, the activation of the hypothalamic-pituitary-adrenal axis by a lipopolysaccharide (endotoxin) is mediated through interleukin-1. Endocrinology 125, 2800-2805 (1989).

78. Ramachandra, R. N., Sehon, A. H. \& Berczi, I. Neuro-hormonal host defence in endotoxin shock. Brain, Behav., Immun. 6, 157-169 (1992).

79. Cavaillon, J. M. \& Adib-Conquy, M. Bench-to-bedside review: endotoxin tolerance as a model of leukocyte reprogramming in sepsis. Crit. Care 10, 233 (2006).

80. Adib-Conquy, M. et al. Up-regulation of MyD88s and SIGIRR, molecules inhibiting Toll-like receptor signaling, in monocytes from septic patients. Crit. Care Med. 34, 2377-2385 (2006)

81. Yoza, B. K. \& McCall, C. E. Facultative heterochromatin formation at the IL-1 beta promoter in LPS tolerance and sepsis. Cytokine 53, 145-152 (2011).

82. Foster, S. L., Hargreaves, D. C. \& Medzhitov, R. Gene-specific control of inflammation by TLR-induced chromatin modifications. Nature 447, 972-978 (2007).

83. Porta, $C$. et al. Tolerance and M2 (alternative) macrophage polarization are related processes orchestrated by p50 nuclear factor kappaB. Proc. Natl Acad. Sci. USA 106, 14978-14983 (2009).

84. Lopez-Collazo, E. \& del Fresno, C. Pathophysiology of endotoxin tolerance: mechanisms and clinical consequences. Crit. Care 17, 242 (2013).

85. Quatrini, L. et al. Host resistance to endotoxic shock requires the neuroendocrine regulation of group 1 innate lymphoid cells. J. Exp. Med. 214, 3531-3541 (2017).

86. Li, C. C., Munitic, I., Mittelstadt, P. R., Castro, E. \& Ashwell, J. D. Suppression of dendritic cell-derived IL-12 by endogenous glucocorticoids is protective in LPSinduced sepsis. PLoS Biol. 13, e1002269 (2015).

87. Sprung, C. L. et al. The effects of high-dose corticosteroids in patients with septic shock. A prospective, controlled study. N. Engl. J. Med. 311, 1137-1143 (1984).

88. Bone, R. C. et al. A controlled clinical trial of high-dose methylprednisolone in the treatment of severe sepsis and septic shock. N. Engl. J. Med. 317, 653-658 (1987).

89. Annane, D. et al. Effect of treatment with low doses of hydrocortisone and fludrocortisone on mortality in patients with septic shock. JAMA 288, 862-871 (2002).

90. Sprung, C. L. et al. Hydrocortisone therapy for patients with septic shock. N. Engl. J. Med. 358, 111-124 (2008).

91. Venkatesh, B. et al. Adjunctive glucocorticoid therapy in patients with septic shock. N. Engl. J. Med. 378, 797-808 (2018).

92. Annane, D. et al. Hydrocortisone plus fludrocortisone for adults with septic shock. N. Engl. J. Med. 378, 809-818 (2018).

93. Rhodes, A. et al. Surviving Sepsis Campaign: International Guidelines for Management of Sepsis and Septic Shock: 2016. Intensive Care Med. 43, 304-377 (2017)
94. Biswas, S. K. \& Lopez-Collazo, E. Endotoxin tolerance: new mechanisms, molecules and clinical significance. Trends Immunol. 30, 475-487 (2009).

95. Brandish, P. E. et al. Development of anti-CD74 antibody-drug conjugates to target glucocorticoids to immune cells. Bioconjugate Chem. 29, 2357-2369 (2018).

96. $\mathrm{Li}, \mathrm{H}$. et al. SARS-CoV-2 and viral sepsis: observations and hypotheses. Lancet 395, 1517-1520 (2020).

97. Huang, C. et al. Clinical features of patients infected with 2019 novel coronavirus in Wuhan, China. Lancet 395, 497-506 (2020).

98. Chen, G. et al. Clinical and immunological features of severe and moderate coronavirus disease 2019. J. Clin. Investig. 130, 2620-2629 (2020).

99. Ledford, H. How does COVID-19 kill? Uncertainty is hampering doctors' ability to choose treatments. Nature 580, 311-312 (2020).

100. Metselaar, J. M., Wauben, M. H., Wagenaar-Hilbers, J. P., Boerman, O. C. \& Storm, G. Complete remission of experimental arthritis by joint targeting of glucocorticoids with long-circulating liposomes. Arthritis Rheum. 48, 2059-2066 (2003).

101. Metselaar, J. M. et al. Liposomal targeting of glucocorticoids to synovial lining cells strongly increases therapeutic benefit in collagen type II arthritis. Ann. Rheum. Dis. 63, 348-353 (2004).

102. Oikarinen, A. \& Autio, P. New aspects of the mechanism of corticosteroidinduced dermal atrophy. Clin. Exp. Dermatol. 16, 416-419 (1991).

103. Oikarinen, A., Haapasaari, K. M., Sutinen, M. \& Tasanen, K. The molecular basis of glucocorticoid-induced skin atrophy: topical glucocorticoid apparently decreases both collagen synthesis and the corresponding collagen mRNA level in human skin in vivo. Br. J. Dermatol. 139, 1106-1110 (1998).

104. Perez, P. et al. Altered skin development and impaired proliferative and inflammatory responses in transgenic mice overexpressing the glucocorticoid receptor. FASEB J. 15, 2030-2032 (2001).

105. Beer, H. D., Fassler, R. \& Werner, S. Glucocorticoid-regulated gene expression during cutaneous wound repair. Vitam. Hormones. 59, 217-239 (2000).

106. Skoner, D. P. et al. Detection of growth suppression in children during treatment with intranasal beclomethasone dipropionate. Pediatrics 105, E23 (2000).

107. Reid, I. R. Glucocorticoid-induced osteoporosis. Bailliere's Best. Pract. Res. Clin. Endocrinol. Metab. 14, 279-298 (2000).

108. Barnes, P. J. \& Adcock, I. M. Glucocorticoid resistance in inflammatory diseases. Lancet 373, 1905-1917 (2009).

109. Schacke, H., Docke, W. D. \& Asadullah, K. Mechanisms involved in the side effects of glucocorticoids. Pharmacol. Therapeutics 96, 23-43 (2002).

110. Schacke, $\mathrm{H}$. et al. Dissociation of transactivation from transrepression by a selective glucocorticoid receptor agonist leads to separation of therapeutic effects from side effects. Proc. Natl Acad. Sci. USA 101, 227-232 (2004).

111. Coghlan, M. J. et al. A novel antiinflammatory maintains glucocorticoid efficacy with reduced side effects. Mol. Endocrinol. 17, 860-869 (2003).

112. Klassen, $C$. et al. Airway epithelial cells are crucial targets of glucocorticoids in a mouse model of allergic asthma. J. Immunol. 199, 48-61 (2017).

113. Obradovic, M. M. S. et al. Glucocorticoids promote breast cancer metastasis. Nature 567, 540-544 (2019).

114. Cui, B. et al. Targeting ROR1 inhibits epithelial-mesenchymal transition and metastasis. Cancer Res. 73, 3649-3660 (2013).

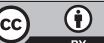

Open Access This article is licensed under a Creative Commons Attribution 4.0 International License, which permits use, sharing, adaptation, distribution and reproduction in any medium or format, as long as you give appropriate credit to the original author(s) and the source, provide a link to the Creative Commons license, and indicate if changes were made. The images or other third party material in this article are included in the article's Creative Commons license, unless indicated otherwise in a credit line to the material. If material is not included in the article's Creative Commons license and your intended use is not permitted by statutory regulation or exceeds the permitted use, you will need to obtain permission directly from the copyright holder. To view a copy of this license, visit http://creativecommons. org/licenses/by/4.0/.

(c) The Author(s) 2020 